\title{
Pragmatic Considerations on Clinical Decision Support from the 2019 Literature
}

\author{
C. Duclos ${ }^{1,2}$, J. Bouaudd ${ }^{3,1}$, Section Editors for the IMIA Yearbook Section on Decision Support \\ 1 Université Sorbonne Paris Nord, Sorbonne Université, INSERM, UMR_ S 1142, LIMICS, Paris, France \\ 2 AP-HP, Hôpital Avicenne, Bobigny, France \\ ${ }^{3}$ AP-HP, Delegation for Clinical Research and Innovation, Paris, France
}

\section{Summary}

Objectives: To summarize significant research contributions published in 2019 in the field of computerized clinical decision support and select the best papers for the Decision Support section of the International Medical Informatics Association (IMIA) Yearbook.

Methods: Two bibliographic databases were searched for papers referring to clinical decision support systems (CDSSS) and computerized provider order entry (CPOE) systems. From search results, section editors established a list of candidate best papers, which were then peer-reviewed by external reviewers. The IMIA Yearbook editorial committee finally selected the best papers on the basis of all reviews including the section editors' evaluation. Results: A total of 1,378 articles were retrieved. Fifteen best paper candidates were selected, the reviews of which resulted in the selection of three best papers. One paper reports on a guideline modeling approach based on clinical decision trees, both clinically interpretable and suitable for implementation in CDSSS. In another paper, authors promote the use of extended Timed Transition Diagrams in CDSSs to formalize consistently recurrent medical processes for chronic diseases management. The third paper proposes a conceptual framework and a grid for assessing the performance of predictive tools based on the critical appraisal of published evidence.

Conclusions: As showed by the number and the variety of works related to decision support, research in the field is very active.

This year's selection highlighted pragmatic works that promote transparency and trust required by decision support tools.

\section{Keywords}

Medical informatics; International Medical Informatics

Association; Yearbook; Decision Support Systems

Yearb Med Inform 2020:155-8

http://dx.doi.org/10.1055/s-0040-1702016

\section{Introduction}

This paper serves as the synopsis of the decision support section of the International Medical Informatics Association (IMIA) Yearbook. It complements the survey paper authored by Jankovic and Chen [1] where the authors seek to identify the features of clinical decision support systems (CDSSs) that may contribute to the observed clinician burnout. The aim of the synopsis is to summarize recent research in the domain of decision support and to select the best papers published in this field in 2019. This literature review focused on research works related to CDSSs and computerized provider order entry (CPOE) systems.

The synopsis is organized as follows: the next section summarizes the process for selecting the best papers on the decision support topic; the following section presents the results of this year's selection process, and the last section comments the contributions of the three best papers, as well as noticeable research works in the field identified during the whole process.

\section{Paper Selection Method}

A comprehensive literature search on topics related to CDSSs and CPOE systems was performed to identify candidate best papers in two bibliographic databases, the PubMed/ Medline database (from the US National Center for Biotechnology Information) and the Web of Science ${ }^{\circledR}$ (WoS, from Clarivate Analytics). PubMed is centered on the biomedical and life sciences literature whereas WoS covers a wider scope of all scientific domains, including biomedicine and life sciences. Both databases were searched with similar queries, tailored to the specificities of each one, targeting journal articles published in 2019, written in English, and related to the aforementioned topics. The adopted strategy was the same as that used in prior years [2] and is based on four exclusive queries that return four disjoint citation subsets. The first query $\left(Q_{\text {pub plain }}\right)$ is based on a plain-text search in PubMed titles and abstracts using keywords. The second query $\left(\mathrm{Q}_{\text {Pub_indexed }}\right)$ relies on the PubMed indexing scheme using $\mathrm{MeSH}$ terms and results are made exclusive of the previous set. The third one $\left(\mathrm{Q}_{\text {WoS re- }}\right.$ stricted) is based on a plain-text search in WoS restricted to the two research areas "Medical Informatics" and "Health Care Sciences \& Services". The fourth query $\left(\mathrm{Q}_{\text {WoS_ filtered }}\right)$ is based on the same plain-text search used in WoS but filtered by non-relevant research areas (e.g., Archeology, Dance, Zoology, etc.) and the two research areas of the previous query. It is of note that the two WoS queries select only non-PubMed-indexed papers that are supposed to be caught by the two PubMed queries.

A first review of the four subsets of retrieved citations was performed by the two section editors to select 15 candidate best papers. Following the IMIA Yearbook protocol, these candidate best papers were then individually reviewed and rated by both section editors, the chief editor of the Decision Support section, and external reviewers from the international Medical Informatics community. Based on the reviewers' ratings and comments, the Yearbook editorial committee then selected the best papers of the year in the decision support domain. 


\section{Review Results}

The 2019 literature search has been performed on January 13, 2020. A total of 1,378 unique references were obtained, distributed as follows: 1,113 for $\mathrm{Q}_{\text {Pub_plain }}, 130$ for $\mathrm{Q}_{\text {Pub_in- }}$

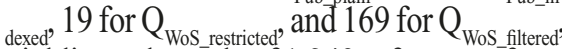
yielding sub-totals of 1,243 references from PubMed, and 188 from WoS. Compared to the previous year, the global query retrieved 230 more papers. After a first individual screening independently performed by both section editors based on the title and abstract of papers, 115 (not rejected by both section editors) were discussed by the two editors to achieve a final selection of 15 candidate best papers. After the external review of these 15 articles, the editorial committee finally selected three of them as best papers for 2019 [3-5] (Table 1). They are discussed in the next section, and summaries of their contents are available in the Appendix.

\section{Discussion and Outlook}

In the first paper, Hendriks et al., [3] propose an approach to the modeling of clinical practice guidelines, which certainly builds on already existing approaches, but which is systematically conducted in order to be scalable and used to represent complex guidelines. They promote the formalism of clinical decision trees (CDTs) as they are both clinically interpretable by healthcare professionals and computer-interpretable, thus suitable for implementation in data-driven CDSSs. The disambiguation of textual guidelines is supported first by the formal, unequivocal, specification of data items used as decision criteria using international coding systems to enforce interoperability, and second by the representation of guideline knowledge as CDTs. The method is applied to the Dutch breast cancer guidelines. Sixty CDTs were built, involving a total of 114 data items, among which, 11 could not be linked to standard terminologies. The authors report the ambiguity of certain criteria, which could be subjective or had multiple definitions. The resulting knowledge base was implemented in a decision support application where it can be interactively browsed or automatically executed. By modeling guidelines in such a way, this work is a step forward in the sharing of encoded knowledge.

In the second paper, Kamišalić et al., [4] tackled the issues linked to the formalization of the medical processes used for managing chronic diseases and their execution in CDSSs. They analyzed the decision-making dimensions of the therapeutic management of chronic diseases, like those known to increase the cardio-vascular risk, and identified three basic levels: therapy strategy, dosage adaptation, and intolerance management. To handle these different aspects consistently, they propose a formalism called extended Timed Transition Diagram (eTTD). With eTTDs, they illustrate the multilevel and fine-grained modeling required to capture the contents of arterial hypertension management guidelines. This detailed demonstration on how procedural knowledge for hypertension management can be formalized to develop a CDSS could certainly be used in other medical domains.

The third paper by Khalifa et al., [5] presents a conceptual and practical framework to help assess confidence in predictive tools. GRASP, for Grade and Assess Predictive Tools, is both a method to look for evidence from the published literature and an analysis grid. It standardizes the assessment of the available literature associated to a predictive tool and the grading of its level of proof. Three phases of evaluation are considered: (i) before the implementation of the tool to assess both its internal and external validity, (ii) during the implementation to assess its potential effect and usability, and (iii) after the implementation to assess its effectiveness and safety. In each phase, the level of evidence can be assessed from the study design. A qualitative conclusion summarizes the direction of evidence (positive, negative, mixed). This grid can be considered as similar to existing grids, for instance the $\mathrm{CON}$ SORT statement for clinical trials. However, it gives a rigorous methodology for a critical appraisal of predictive tools and could be extended to all kind of CDSSs. It might be a useful tool to extend the evidence-based culture in the field of medical informatics.

Besides the three best papers selected for the Decision Support section of the 2020 edition of the IMIA Yearbook, several other works retrieved from the literature review deserve to be cited. Some of them deal with the personalization of decisions. Laleci et al., [6] propose a scientific and technical approach to develop personalized care plans that comply with clinical practice guidelines for the management of complex polypathology situations. Jafarpour et al., [7] propose a solution to dynamically manage the conflicts that can rise in this type of complex contexts. Ben Souissi et al., [8] introduce the use of health information technology involving multiple criteria decision to support the choice between antibiotics alternatives. Interestingly, other works promote the creation and sharing of operational knowledge bases as exemplified by Hendriks et al., [3]. Thus, Huibers et al., [9] transform the textual STOPP/START criteria into unambiguous definitions mapped to medical terminologies. Canovas et al., [10] formalize EUCAST expert rules as an ontology and production rules to detect antimicrobial therapies at risk of failure. Müller et al., [11] propose an open diagnostic knowledge base that can compete with commercial ones. Replacing humans is another topic of research and Spänig et al., [12] work on two aspects to virtualize a doctor: the automatic acquisition of data through sensors and speech recognition, and the automation of diagnostic reasoning. Rozenblum et al.,[13] propose a machine learning method to generate clinically valid alerts to detect errors in prescriptions.

Acceptability of CDSS is another key point. Kannan et al., [14] propose a method for a CDSS design to best meet a precisely specified and assessable user purpose. Design alerts may also avoid rejection of CDSSs by caregivers. Fernandes et al., [15] created algorithms able to aggregate, filter, and reduce the notifications delivered to healthcare professionals. Amrose et al., [16] tried to understand in real life the impact of alerts on users and to find the actions they triggered. Finally, it is always interesting to obtain varied evaluation results of controversial CDSSs. In this respect, Kim et al., [17] evaluated Watson for Oncology in thyroid carcinoma and reported a concordance rate with local practices considered as too low to adopt the tool.

As evidenced by the number and the variety of works around decision support, research in the field is very active. This year's selection highlighted pragmatic works that promote the transparency and sharing of the 
Table 1 Best paper selection of articles for the IMIA Yearbook of Medical Informatics 2020 in the section 'Decision Support'. The articles are listed in alphabetical order of the first author's surname.

\section{Section}

Decision Support

- Hendriks MP, Verbeek XAAM, van Vegchel T, van der Sangen MJC, Strobbe LA, Merkus JWS, Zonderland HM, Smorenburg CH, Jager A, Siesling S. Transformation of the National Breast Cancer Guideline Into Data-Driven Clinical Decision Trees. JCO Clin Cancer Inform 2019;3:1-14.

- Kamišalić A, Riaño D, Kert S, Welzer T, Nemec Zlatolas L. Multi-level medical knowledge formalization to support medical practice for chronic diseases. Data \& Knowledge Engineering 2019;119:36-57.

- Khalifa M, Magrabi F, Gallego B. Developing a framework for evidence-based grading and assessment of predictive tools for clinical decision support. BMC Med Inform Decis Mak 2019;19(1):207.

knowledge bases used by decision support tools, as well as the grading of their utility. The ultimate goal is that users could trust such tools to, then, use them.

\section{Acknowledgement}

We would like to thank all the present and past editorial boards of the IMIA Yearbook, especially Martina Hutter and Adrien Ugon for their support, as well as the reviewers for their participation to the selection of the best papers for the Decision Support section. We cannot end this synopsis without a meaningful thought for our colleague and friend Vassilis Koutkias who started, this year again, to tackle the tasks of a Decision Support section co-editor, but passed away in last December, and unfortunately could not finish.

\section{References}

1. Jankovic I, Chen JH. Clinical Decision Support and Implications for the Clinician Burnout Crisis. Yearb Med Inform 2020:145-54.

2. Koutkias V, Bouaud J. Contributions on Clinical Decision Support from the 2018 Literature. Yearb Med Inform 2019 Aug;28(1):135-7.

3. Hendriks MP, Verbeek XAAM, van Vegchel T, van der Sangen MJC, Strobbe LJA, Merkus JWS, et al. Transformation of the National Breast Cancer Guideline Into Data-Driven Clinical Decision Trees. JCO Clin Cancer Inform. 2019;3:1-14.

4. Kamišalić A, Riaño D, Kert S, Welzer T, Nemec Zlatolas L. Multi-level medical knowledge formalization to support medical practice for chronic diseases. Data \& Knowledge Engineering. 2019;119:36-57.

5. Khalifa M, Magrabi F, Gallego B. Developing a framework for evidence-based grading and assessment of predictive tools for clinical decision support. BMC Med Inform Decis Mak 2019;19(1):207.

6. Laleci GB, Yuksel M, Sarigul B, Arvanitis TN, Lindman P, Chen R, et al. A Collaborative Platform for Management of Chronic Diseases via Guideline-Driven Individualized Care Plans. Comput Struct Biotechnol J 2019;17:869-85.

7. Jafarpour B, Raza Abidi S, Van Woensel W, Raza Abidi SS. Execution-time integration of clinical practice guidelines to provide decision support for comorbid conditions. Artif Intell Med 2019;94:117-37.

8. Ben Souissi S, Abed M, El Hiki L, Fortemps P, Pirlot M. PARS, a system combining semantic technologies with multiple criteria decision aiding for supporting antibiotic prescriptions. J Biomed Inform 2019:99:103304.

9. Huibers CJA, Sallevelt BTGM, de Groot DA, Boer MJ, van Campen JPCM, Davids CJ, et al. Conversion of STOPP/START version 2 into coded algorithms for software implementation: A multidisciplinary consensus procedure. Int J Med
Inform 2019;125:110-7.

10. Cánovas-Segura B, Morales A, Juarez JM, Campos $\mathrm{M}$, Palacios F. Impact of expert knowledge on the detection of patients at risk of antimicrobial therapy failure by clinical decision support systems. J Biomed Inform 2019;94:103200.

11. Müller L, Gangadharaiah R, Klein SC, Perry J, Bernstein G, Nurkse D, et al. An open access medical knowledge base for community driven diagnostic decision support system development. BMC Med Inform Decis Mak 2019;19(1):93

12. Spänig S, Emberger-Klein A, Sowa JP, Canbay A, Menrad K, Heider D. The virtual doctor: An interactive clinical-decision-support system based on deep learning for non-invasive prediction of diabetes. Artif Intell Med 2019;100:101706.

13. Rozenblum R, Rodriguez-Monguio R, Volk LA, Forsythe KJ, Myers S, McGurrin M, et al. Using a Machine Learning System to Identify and Prevent Medication Prescribing Errors: A Clinical and Cost Analysis Evaluation. Jt Comm J Qual Patient Saf 2020;46(1):3-10.

14. Kannan V, Basit MA, Bajaj P, Carrington AR, Donahue IB, Flahaven EL, et al. User stories as lightweight requirements for agile clinical decision support development. J Am Med Inform Assoc 2019;26(11):1344-54.

15. Fernandes CO, Miles S, Lucena CJP, Cowan D. Artificial Intelligence Technologies for Coping with Alarm Fatigue in Hospital Environments Because of Sensory Overload: Algorithm Development and Validation. J Med Internet Res 2019;21(11):e15406.

16. Amroze A, Field TS, Fouayzi H, Sundaresan D, Burns L, Garber L, et al. Use of Electronic Health Record Access and Audit Logs to Identify Physician Actions Following Noninterruptive Alert Opening: Descriptive Study. JMIR Med Inform 2019;7(1):e12650.

17. Kim M, Kim BH, Kim JM, Kim EH, Kim K, Pak $\mathrm{K}$, et al. Concordance in postsurgical radioactive iodine therapy recommendations between Watson for Oncology and clinical practice in patients with differentiated thyroid carcinoma. Cancer 2019;125(16):2803-9.

\section{Correspondence to:}

Pr. Catherine Duclos

LIMICS INSERM 1142

Faculté Léonard de Vinci

74 rue Marcel Cachin, 93017 Bobigny, France

E-mail: catherine.duclos@aphp.fr 
Appendix: Content Summaries of Best Papers for the Decision Support Section of the 2020 IMIA Yearbook

\section{Hendriks MP, Verbeek XAAM, van Vegchel T, van der Sangen MJC, Strobbe LJA, Merkus JWS, Zonderland HM, Smorenburg CH, Jager $A$, Siesling $S$ \\ Transformation of the National Breast Cancer Guideline into data-driven clinical decision trees}

\section{JCO Clin Cancer Inform 2019 May;3:1-14}

Since clinical practice guidelines are still narrative and described in large textual documents, the aim of this work was to model complex guidelines as data-driven clinical decision trees (CDTs) that could be still human-interpretable while computer-interpretable for implementation in decision support systems. The Dutch national breast cancer guidelines were translated into CDTs. Data items, which characterize the patient and the tumor and represent decisional criteria, were encoded unambiguously using existing classifications and coding systems related to breast cancer when feasible. In total, 60 CDTs were necessary to cover the whole guidelines, driven by 114 data items. Of all data items, $101(89 \%)$ could be coded using existing classification and coding systems. All 60 CDTs represented 376 unique patient subpopulations. Complex guidelines could be transformed as systematically constructed modular data-driven CDTs that are clinically interpretable and executable in a decision support application.

\section{Kamišalić A, Riaño D, Kert S, Welzer T, Nemec Zlatolas L \\ Multi-level medical knowledge formalization to support medical practice for chronic diseases}

\section{Data \& Knowledge Engineering 2019;} 119:36-57

This research is focused on knowledge representation to support the medical processes involved in chronic diseases management, which can be viewed as a procedural and sequential application of knowledge. An intuitive, easy, and effective mechanism for medical knowledge formalization is proposed through a formalism called extended Timed Transition Diagram (eTTD). This formalism allows for the consistent representation of three basic levels of decision making that should be taken into account in the prescription and adaptation of longterm treatment: therapy strategy, dosage, and intolerances. The methodology can be manually applied to build eTTDs from clinical practice guidelines. eTTDs implementation is demonstrated by modeling clinical prac- tice guidelines for the therapeutic management of arterial hypertension. The obtained models can be used as a baseline framework for the development of decision support systems involving medical procedures.

\section{Khalifa M, Magrabi F, Gallego B}

Developing a framework for evidencebased grading and assessment of predictive tools for clinical decision support

\section{BMC Med Inform Decis Mak 2019 Oct 29;19(1):207}

Deciding to choose a clinical predictive tool in clinical practice should be guided by its correctly assessed effectiveness. The objective of this work is to developp a conceptual and practical framework to Grade and Assess Predictive tools (GRASP) and provide clinicians with a standardised, evidence-based system to support their search for and selection of efficient predictive tools. The GRASP framework grades predictive tools based on published evidence across three dimensions: phase of evaluation, level of evidence, and direction of evidence. The final grade of the tool is based on the phase of evaluation that gets the hightest grade, supported by the highest level of positive or mixed evidence that supports a positive conclusion. This framework was successfully applied to five predictive tools. GRASP report updates could be a way to maintain a data base that documents the evidence of predictive tools. 